\title{
Starch/Banana Pseudostem Biocomposite Films for Potential Food Packaging Applications
}

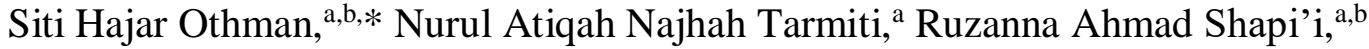 \\ Siti Mariam Mohd Zahiruddin, ${ }^{\mathrm{a}}$ Intan Syafinaz Mohammed Amin Tawakkal, and \\ Roseliza Kadir Basha ${ }^{a}$
}

\begin{abstract}
Banana pseudostems (BP) are a promising cellulose source that remains underutilized despite its potential to be formed as biodegradable food packaging material. This work was directed towards utilizing BP waste in a powder form to produce potential food packaging material that is environmentally friendly. A solvent casting method was used to produce starch/BP films at different BP percentage compositions $(0,10,20,30$, and $40 \mathrm{wt} \%$ on the dry basis of starch). The effects of different percentage compositions of BP in starch on the morphology, optical, mechanical, and barrier properties of the films were investigated. The optical and mechanical properties of the starch films decreased with the addition of BP. However, the mechanical and barrier properties of the films improved with the increase in BP composition. An optimum percentage composition of $40 \mathrm{wt} \%$ BP powder can be utilized to produce starch/BP films for potential food packaging application.
\end{abstract}

Keywords: Banana pseudo-stem; Film; Food packaging; Starch; Solvent casting

Contact information: a: Department of Process and Food Engineering, Faculty of Engineering, Universiti Putra Malaysia, 43400 Serdang, Selangor, Malaysia; b: Materials Processing and Technology Laboratory, Institute of Advanced Technology, Universiti Putra Malaysia, 43400 Serdang, Selangor, Malaysia;

* Corresponding author: s.hajar@upm.edu.my

\section{INTRODUCTION}

Banana crops produce a large volume of agricultural byproducts every year. Each banana plant produces only one bunch of bananas and then it cannot be used for the next harvest. After harvesting, the bare banana pseudostems (BP) are usually cut and left in the plantation soil to be converted into organic material; however, this can potentially become an environmental issue. Banana pseudostems form a major waste material in large-scale farming operations, and their disposal has become a huge problem if the proper agricultural waste management is not practiced. Nonetheless, BP have the potential to be converted into profitable products.

Banana pseudostems are a promising cellulose source that remains underutilized. Banana pseudostems are fibrous in nature and high in cellulose content (Pappu et al. 2015). The high cellulose content in BP makes BP a promising material to use in environmentally friendly food packaging materials. Apart from that, BP exhibits favorable properties such as edibility, biocompatibility, non-toxicity, and a low cost. However, BP alone cannot be used to produce food packaging material due to its fibrous nature. The addition of other biopolymers, such as starch, may produce biodegradable food packaging material.

Starch is one of the most promising bio-based packaging materials because starch is a renewable source, easy to handle, low in cost, and readily available in the current 
marketplace (Resa et al. 2012; Othman 2014). Starch films have also been found to be transparent, odorless, and colorless, which will allow the production of a good packaging material (Jiménez et al. 2012). Starch can be mainly found in the tuber plant and maize plant. The main components of starch granules consist of linear amylose and highly branched amylopectin that is formed by the glucose units via $\alpha-1,4$ bonds (Ji et al. 2017). Both amylose and amylopectin exhibit different properties, whereby amylose is stronger than amylopectin due to its high crystallinity compared to amylopectin.

Although the native starch alone can be used to produce a biodegradable food packaging film, the film will usually exhibit poor mechanical and barrier properties (Othman et al. 2019a; Othman et al. 2019b; Shapi'i et al. 2019; Zahiruddin et al. 2019). One of the effective ways to overcome this limitation is by incorporating other biopolymers with the starch film, thus producing a biocomposite film. Generally, the combination of biopolymers with other biopolymers (biocomposite) has been widely used to improve the properties of the prepared films (Vásconez et al. 2009). Cellulose, which is contained in BP, appears to be a potential biopolymer that can be incorporated with starch due to its superior mechanical properties. Cellulose can be added to starch film to enhance the mechanical properties and barrier resistance of the film (Ferracane et al. 2014; Nordin et al. 2018; Othman et al. 2019b). Cellulose has proven to be promising as a biocomposite material in film packaging and shows satisfactory physical properties, such as a relatively high tensile strength, high stiffness, and a high Young's modulus (Zainuddin et al. 2013).

A glance through the literature reveals that films containing cellulose have been developed for food packaging application. The potential use of cellulose nanofibril (CNF) as a reinforcing agent in banana starch-based films was investigated by Tibolla et al. (2019). CNF material was prepared from banana peel by enzymatic hydrolysis method and was utilized to produce banana starch-based nanocomposite films via the casting method. They reported that the films exhibited a remarkable improvement on mechanical and water barrier properties, opacity, and UV light barrier compared to the control film. Meanwhile, Pacaphol et al. (2019) produced nanocellulose suspensions and coated the suspensions on spinach leaves to extend the shelf life of spinach during postharvest storage. It was found that the coated leaves with 0.3 and $0.5 \% \mathrm{w} / \mathrm{v}$ nanocellulose concentrations maintained the appearance, chlorophyll, color, and moisture content of the leaves after 3 days storage at $25{ }^{\circ} \mathrm{C}$ which was much better compared to uncoated leaves. On the other hand, Wang et al. (2020) developed silver nanoparticles/polyvinyl alcohol/bacterial nanocellulose packaging films and investigated the application of the films on raw beef. They found that the films were able to extend the shelf life of raw beef. It is worth mentioning that cellulose offers a great potential as reinforcing and active material for biopolymers films food packaging application.

Nevertheless, the percentage composition of the biopolymers in the biocomposite is an imperative parameter that needs to be determined to produce efficient food packaging material. The properties of the food packaging material are largely dependent on the percentage composition of the biocomposite. To the best of the authors' knowledge, no studies have been conducted to produce and characterize starch/BP biocomposite films at different percentage composition for food packaging application. Thus, this work is directed to investigate the effects of different percentage composition of $\mathrm{BP}(0,10,20,30$, and $40 \mathrm{wt} \%$ ) to starch on the morphological, optical, mechanical, and barrier properties of the biocomposite film. This work is also important to determine the maximum percentage composition that can be utilized to produce food packaging material to reduce agricultural waste from banana plantation. 


\section{EXPERIMENTAL}

\section{Materials}

All the materials used in this work were food grade, edible, and non-toxic. Tapioca starch (food grade) was obtained from LGC Scientific Sdn. Bhd (Wilayah Persekutuan, Malaysia). The amylose content of tapioca starch is within the range of $16 \%$ to $18 \%$ and the remainder is amylopectin (Wheatley 2003). Banana pseudostems (Musa acuminata $\times$ balbisiana ABB cv. Awak) were obtained from a local farm located at Lentang, Pahang, Malaysia. The major chemical contents in BP are cellulose $(55.5 \mathrm{wt} \%)$, hemicellulose $(5.4$ $\mathrm{wt} \%)$, lignin (22.3 wt\%), pectin (5.7 wt\%), wax (1.44 wt\%), and extractives (7.6 wt \%) (Pappu et al. 2015).

\section{Preparation of BP Powder}

The inner part of BP (Fig. 1a) was sliced manually using a sterile knife. The BP slices (Fig. 1b) were then rinsed with running tap water followed by rinsing with distilled water and dried in a cabinet dryer (Lab Companion; Jeio Tech Co., Ltd., Seoul, Korea) at $60{ }^{\circ} \mathrm{C}$ for 2 days. The dried BP slices (Fig. 1c) were then milled (Model: Boma LG-LJ618; I Retailers Pvt. Ltd., Delhi, India) at 15,000 rpm and sieved with mesh pore size of $0.2 \mathrm{~mm}$ to obtain fine BP powders (Fig. 1d). The powder was kept in a desiccator (temperature: 25 ${ }^{\circ} \mathrm{C}$, relative humidity (RH): 53\%) prior to further work. Saturated magnesium nitrate was utilized to provide the constant RH of $53 \%$ inside the desiccator.

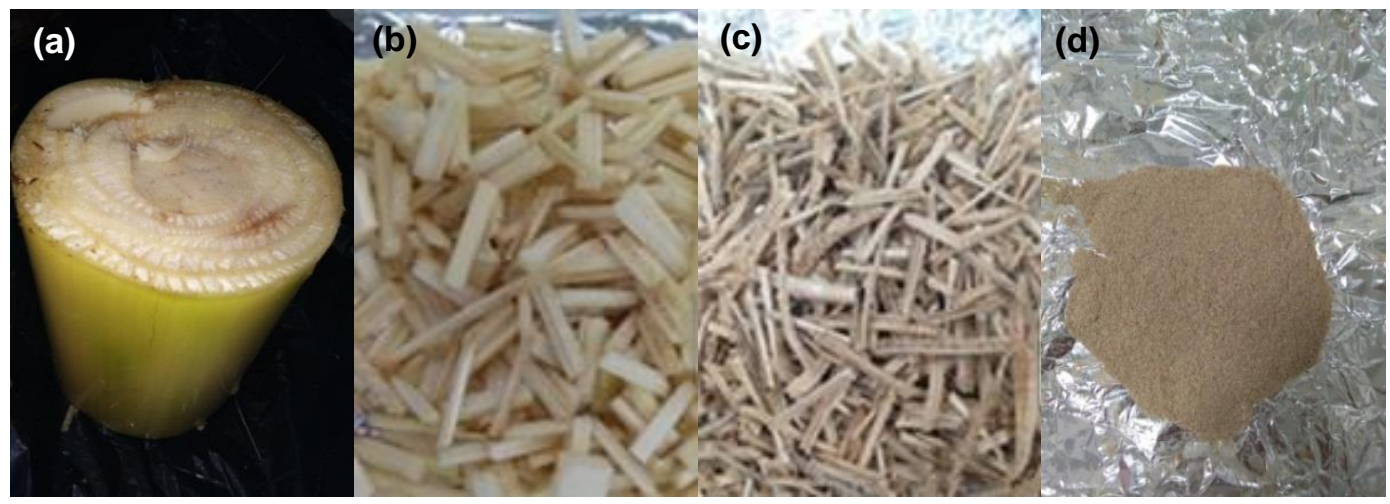

Fig. 1. Images of (a) BP, (b) BP slices, (c) dried BP slices, and (d) BP powder

\section{Preparation of neat starch and starch/BP films}

An amount of $3 \mathrm{~g}$ of tapioca starch was added into $100 \mathrm{~mL}$ of distilled water to obtain a film forming solution. The mixture was stirred using a stirrer hot plate (Model HS0707V2; Favorit, Chicago, IL, USA) with continuous stirring for $5 \mathrm{~min}$. Then, a designated amount of $\mathrm{BP}(0,10,20,30$, and $40 \mathrm{wt} \%$ on the dry basis of the tapioca starch) was dispersed in the starch mixture. The mixture was stirred continuously and heated until the solution gelatinized at $70{ }^{\circ} \mathrm{C}$. An amount of $30 \mathrm{~mL}$ solution mixture was then spread evenly in a petri dish $\left(14-\mathrm{cm}\right.$ diameter) and dried at room temperature $\left(25^{\circ} \mathrm{C}\right)$ for $48 \mathrm{~h}$. After drying, the films were peeled off and kept in a desiccator (temperature: $25^{\circ} \mathrm{C}, \mathrm{RH}$ : $53 \%$ ) prior to the characterization work. 


\section{Methods}

Morphological properties

The morphology of starch/BP films was observed using scanning electron microscopy (SEM) (Model: S-3400N; Hitachi, Tokyo, Japan). The films were coated with gold sputter coater (Model K575X; Emitech, Kent, United Kingdom) before being observed via SEM. The test was conducted at the voltage of $15 \mathrm{kV}$.

\section{Optical properties}

The color and transparency of the films were determined using the ASTM D1746 (2015) standard. Both analyses were performed using a color spectrophotometer (Model: UltraScan PRO; HunterLab, Reston, VA, USA). The films were cut into $100 \mathrm{~mm} \times 15 \mathrm{~mm}$ and placed directly in the spectrophotometer test cell. Color analysis was determined using the International Commission on Illumination (CIELAB) color system with color parameters of $L^{*}, a^{*}$, and $b^{*}$, where $L^{*}=$ Lightness $(0=$ black to $100=$ white $) ; a^{*}=$ green $(-60)$ to red $(+60)$; and $b^{*}=$ blue (-60) to yellow (+60). Transparency was determined by measuring the light absorption of the films at the wavelength of visible light at $600 \mathrm{~nm}$ using the color spectrophotometer.

The total color difference $\left(\Delta E^{*}\right)$ of the starch/BP films was calculated according to the following formula:

$$
\Delta E=\sqrt{ }\left(\left(\Delta L^{*}\right)^{2}+\left(\Delta a^{*}\right)^{2}+\left(\Delta b^{*}\right)^{2}\right)
$$

\section{Mechanical properties}

A texture analyzer (Model: TA.XT2; Stable Micro Systems, Surrey, UK) was used to investigate the mechanical properties of the films. The film samples were cut into 100 $\mathrm{mm} \times 15 \mathrm{~mm}$ using a film specimen cutter according to ASTM D882 (2018). The samples were then clamped between the grips of the texture analyzer and the analysis was run with the clamp distance of $60 \mathrm{~mm}$ and a crosshead speed of $0.5 \mathrm{~mm} / \mathrm{s}$. The mechanical properties including the tensile strength (TS, maximum force used during measurement), elongation at break (EAB, ratio of elongation to original to the length of the sample), and Young's modulus (YM) of elasticity of the films were determined.

\section{Barrier properties}

Barrier properties of the films were investigated in terms of water vapor and oxygen gas permeability. The thickness of the films was measured using a micrometer (Model MDC-1" SX; Mitutoyo, Kanagawa, Japan), and the average thickness of the films was found to be $0.10 \mathrm{~mm}$. Note that the thickness of the films produced in this work were not significantly difference for different percentage compositions of BP powder.

The water vapor barrier analysis was performed by determining the water vapor permeability of the film according to the JIS Z 0208 (2017) standard. The films were cut (shape: circle, diameter: $7 \mathrm{~cm}$ ) and placed on a cup (No. 318 water permeability cup, Yasuda Seiki Seisakusho Ltd., Hyogo, Japan) containing $10 \mathrm{~g}$ anhydrous calcium chloride $(\mathrm{RH}=0 \%)$. The films were sealed to the cup base with a ring using a mixture of paraffin and bee wax (ratio 4:1). Each cup was placed in a desiccator (temperature: $25^{\circ} \mathrm{C}, \mathrm{RH}$ : $53 \%$ ). Changes of the weight of the cup were determined periodically every $24 \mathrm{~h}$ for 10 days and plotted to obtain the weight changes versus time graph. Water vapor permeability (WVP) was calculated using the following formulas,

$$
\text { Water vapor transmission rate }(\mathrm{WVTR})=(w / t) / A
$$


Water vapor permeability $($ WVP $)=W V T R \times h / \Delta P$

where $w / t$ is the slope of weight changes versus time graph $(\mathrm{g} / \mathrm{h}), A$ is the transmission area of the film $\left(\mathrm{m}^{2}\right), h$ is the thickness of the film (m), and $\Delta P$ is the difference of partial pressures $(\mathrm{Pa})$.

An oxygen permeation system equipped with a calorimetric sensor (Model: OXTRAN 1/50; MOCON Inc., Minneapolis, MN, USA) was used to determine the oxygen permeability of the films. The analysis was conducted at a pressure of 3 psi according to ASTM F1927 (2014). The oxygen transmission rate (OTR) was measured for circular films (surface area: $50 \mathrm{~cm}^{2}$ ). During testing, the film was placed in the chamber and clamped. Oxygen was introduced into the upper half of the sample chamber while nitrogen gas was injected into the lower half of the chamber. The oxygen barrier property was quantified by the oxygen permeability $(\mathrm{OP})$, which indicates the amount of oxygen that permeates per unit of area and time. Oxygen permeability correlates with OTR by the following equation:

$$
O P=O T R \times h
$$

\section{Statistical analysis}

Statistical analysis of the obtained experimental data was performed using Minitab software (Minitab Inc., version 16, State College, PA, USA). Data were represented as a mean value \pm standard deviation of a triplicate of each measurement $(n=3)$. A significant difference between means was evaluated using the analysis of variance (ANOVA). The Tukey test was used for comparing mean values and differences between means were considered significant when $\mathrm{p}<0.05$.

\section{RESULTS AND DISCUSSION}

\section{Morphological Properties}

Figures $2 \mathrm{a}, \mathrm{b}$, and $\mathrm{c}$ show the morphology of the BP powder, pure starch film, and starch/BP film, respectively. Figure 2a shows different shapes and sizes of BP particles when observed at 300× magnification. Flake-like shapes, fibrous shapes, and granular shapes of different particles were observed from the SEM image. The particles were randomly positioned, and the different shapes and sizes of the particles were due to the different components that existed in BP powder, which include cellulose, hemicellulose, lignin, pectin, wax, and extractives (Pappu et al. 2015). The SEM image of neat starch film (Fig. 2b) shows a relatively smooth morphology of the film. This finding was supported by Wittaya (2009) who reported smooth surface morphology of the neat starch film.

When comparing the SEM images of the neat starch film (Fig. 2b) and starch film incorporated with $40 \mathrm{wt} \%$ BP (Fig. 2c), as expected, the $40 \mathrm{wt} \% \mathrm{starch} / \mathrm{BP}$ film became rough with the addition of BP consistent with the finding of Orsuwan et al. (2016) and Faradilla et al. (2017). This was because powder particles were distributed on the surface of the starch film. The difference in sizes and shapes of BP added into the starch film caused an overall poor dispersion and irregular arrangement of BP, thus resulting in the rough surface of BP/starch film (Cheng et al. 2019). The control film shows a smoother, more uniform, and more highly compact surface with no pores.

According to Zhang et al. (2018), the difference in the surface morphology of films is closely associated with the size and morphology of particles added into the film. A more 
similar size and regular morphology of the prepared particles results in a smoother and more uniform surface of the resulting film.
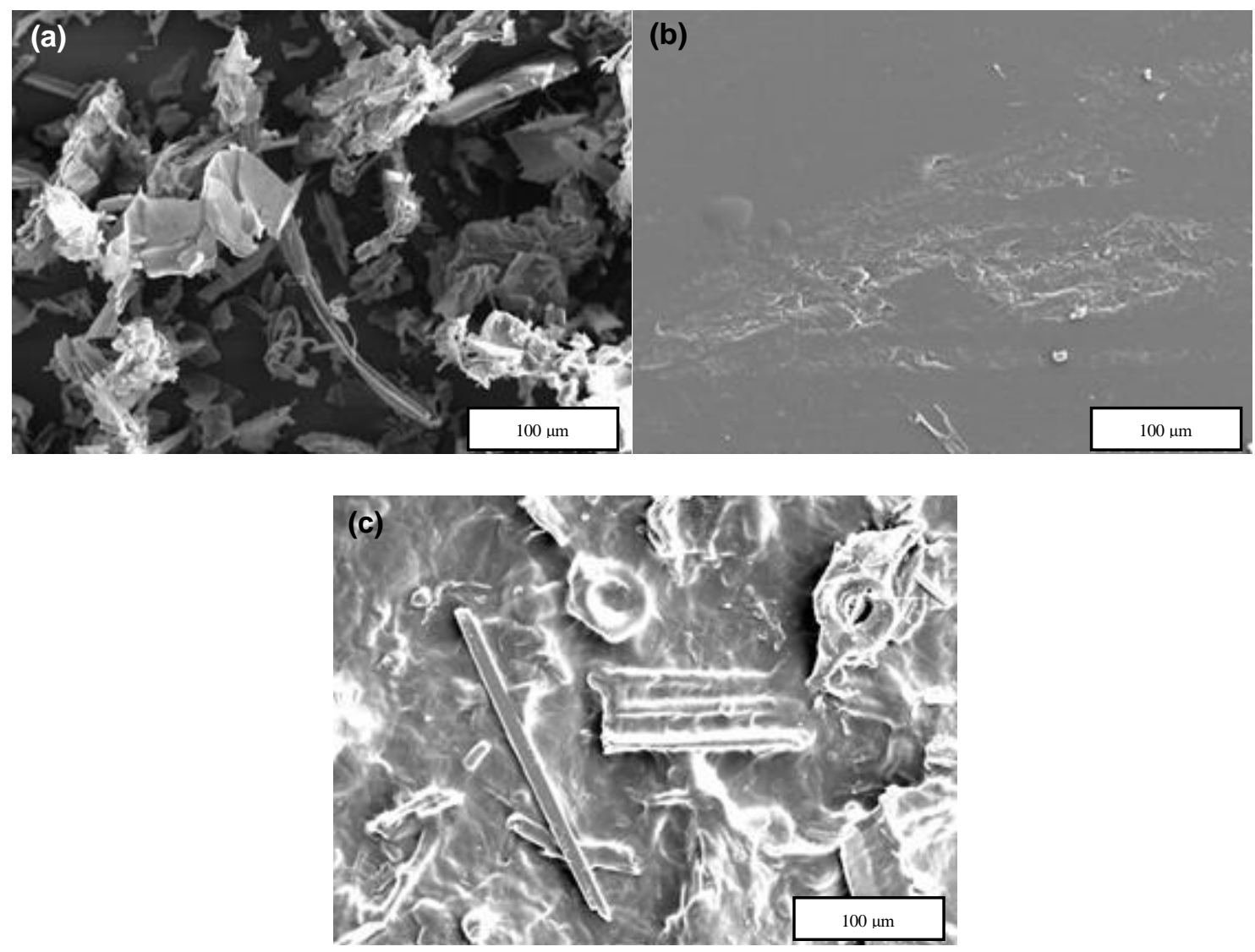

Fig. 2. SEM images at magnification $300 \times$ of (a) BP powder, (b) neat starch film, and (c) starch film incorporated with $40 \mathrm{wt} \% \mathrm{BP}$

\section{Optical Properties}

The color and transparency of food packaging materials are two important factors that will affect the acceptance of consumers toward the packaging material as well as toward the food product wrapped with the film. The optical properties of the neat starch and starch/BP films were characterized in terms of lightness intensity $\left(L^{*}\right)$, redness $\left(a^{*}\right)$, yellowness $\left(b^{*}\right)$, total color changes $(\Delta E)$, and transmittance at $600 \mathrm{~nm}$ as shown in Figs. $3 a$ through e, respectively. For clarity, the data are also listed in Table 1.

Figure 3a shows that starch films added with BP exhibited lower $L^{*}$ values than those of the neat starch film. The decrement became more prominent with the increase in BP composition. Films without the addition of BP exhibited the highest lightness intensity because it was more colorless, translucent, and clearer compared to other films as was observed during film preparation; while films with addition of $40 \mathrm{wt} \% \mathrm{BP}$ exhibited the lowest $L^{*}$ value with approximately $30 \%$ reduction compared to the neat starch film. The increase in the composition of BP that was light brown in color resulted in the reduction of the lightness of the film. 

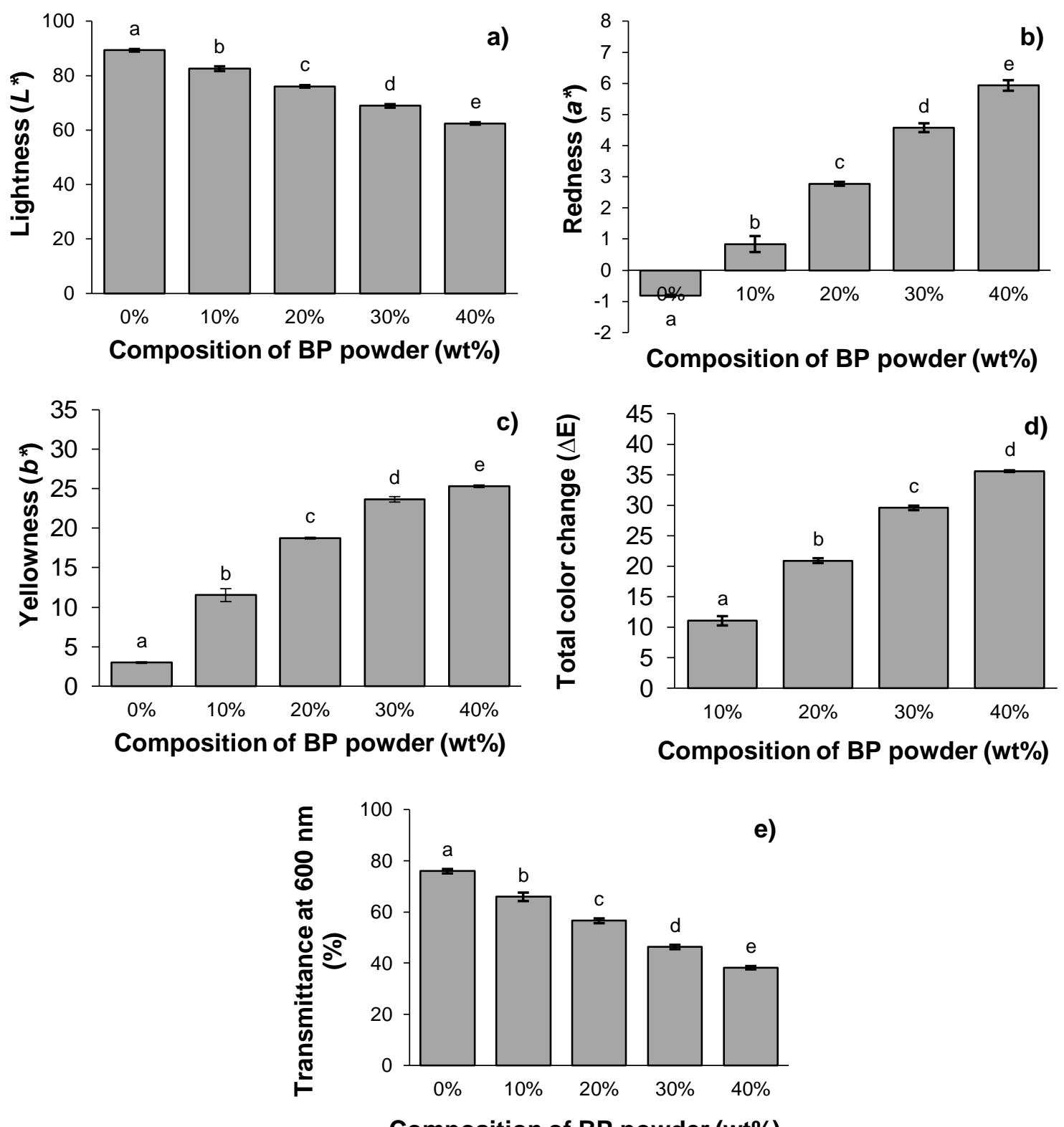

Composition of BP powder (wt\%)

Fig. 3. (a) Lightness intensity, (b) redness, (c) yellowness, (d) total color changes, and (e) transmittance at $600 \mathrm{~nm}$ of the neat starch and starch/BP films. Different letters indicate a statistically significant difference $(p<0.05)$.

Figure $3 \mathrm{~b}$ shows that the neat starch film exhibited a negative $a^{*}$ average value of -0.82 , indicating that the color of the films deviated towards green compared to other films due to the white color of tapioca starch. However, the $a^{*}$ value of the neat starch film was almost zero, indicating that the green tone was almost unperceivable. Figures $3 \mathrm{~b}$ and $\mathrm{c}$ also demonstrate that the $a^{*}$ and $b^{*}$ values increased with the addition of BP. There was a trend of increasing $a^{*}$ and $b^{*}$ values with the increase in BP composition indicating that the color of the starch/BP films deviated towards redness and yellowness, which resulted in a cloudy effect in the films. The increase in the amount of BP in the starch film caused the increase in $a^{*}$ and $b^{*}$ values of the film due to the color of the BP, which was light brown. The addition of other biopolymers into the films increased the cloudiness of the films (Hanpin 
and Stephen 2013), which resulted in a lower overall lightness and explain the increasing $a^{*}$ and $b^{*}$ values of the films.

Table 1. Optical, Mechanical, and Barrier Properties of Starch Films Added with Different Percentage Composition of BP

\begin{tabular}{|c|c|c|c|c|c|}
\hline Properties & \multicolumn{5}{|c|}{ Optical } \\
\hline Sample Films & $L^{*}$ & $a^{*}$ & $\boldsymbol{b}^{\star}$ & $\Delta E$ & $\begin{array}{l}\text { Transmittance } \\
\text { at } 600 \mathrm{~nm}(\%)\end{array}$ \\
\hline 0 & $89.28 \pm 0.80^{a}$ & $-0.82 \pm 0.05^{a}$ & $2.98 \pm 0.17^{a}$ & 0 & $75.90 \pm 1.49^{a}$ \\
\hline 10 & $82.54 \pm 1.49^{b}$ & $0.84 \pm 0.45^{b}$ & $11.53 \pm 1.42^{\mathrm{b}}$ & $11.04 \pm 1.32^{\mathrm{a}}$ & $65.89 \pm 2.86^{b}$ \\
\hline 20 & $76.04 \pm 0.77^{c}$ & $2.78 \pm 0.11^{c}$ & $18.75 \pm 0.19^{c}$ & $20.90 \pm 0.68^{b}$ & $56.52 \pm 1.61^{\mathrm{C}}$ \\
\hline 30 & $68.87 \pm 1.09^{d}$ & $4.58 \pm 0.25^{\mathrm{d}}$ & $23.66 \pm 0.59^{d}$ & $29.54 \pm 0.63^{c}$ & $46.29 \pm 1.46^{\mathrm{d}}$ \\
\hline 40 & $62.43 \pm 0.77^{e}$ & $5.93 \pm 0.29^{\mathrm{e}}$ & $25.31 \pm 0.29^{\mathrm{e}}$ & $35.57 \pm 0.23^{d}$ & $38.19 \pm 1.10^{\mathrm{e}}$ \\
\hline Properties & \multicolumn{5}{|c|}{ Mechanical } \\
\hline Sample Films & \multirow{2}{*}{\multicolumn{2}{|c|}{$\frac{\text { TS (MPa) }}{30.19+100^{\mathrm{a}}}$}} & \multicolumn{2}{|l|}{ YM (MPa) } & EAB (\%) \\
\hline 0 & & & \multirow{2}{*}{\multicolumn{2}{|c|}{$\frac{975.27 \pm 26.34^{\mathrm{a}}}{442.46+45.62^{b}}$}} & $116.35 \pm 0.42^{\mathrm{a}}$ \\
\hline 10 & \multicolumn{2}{|c|}{$16.02 \pm 0.90^{\mathrm{b}}$} & & & $113.44 \pm 0.42^{b}$ \\
\hline 20 & \multicolumn{2}{|c|}{$19.47 \pm 0.35^{c}$} & \multicolumn{2}{|c|}{$547.61 \pm 35.96^{c}$} & \multirow{2}{*}{$112.04 \pm 0.71^{d}$} \\
\hline 30 & \multicolumn{2}{|c|}{$21.14 \pm 0.61^{d}$} & \multicolumn{2}{|c|}{$591.40 \pm 19.12^{\mathrm{d}}$} & \\
\hline 40 & \multirow{2}{*}{\multicolumn{2}{|c|}{$24.20 \pm 1.61^{\mathrm{e}}$}} & $659.69 \pm 30.8$ & & $111.71 \pm 0.44^{d}$ \\
\hline Properties & & & \multicolumn{2}{|c|}{ Barrier } & \\
\hline Sample Films & \multicolumn{2}{|c|}{$\frac{\text { WVP (g.m/m².kPa.h) }}{183 \times 10^{-5 a}}$} & & \multicolumn{2}{|c|}{ OP (cc/m² day.m) } \\
\hline 0 & \multirow{2}{*}{\multicolumn{2}{|c|}{$\frac{1.83 \times 10^{-5 a}}{1.22 \times 10^{-5 b}}$}} & & \multicolumn{2}{|c|}{$6.01 \times 10^{5 a}$} \\
\hline 10 & & & & \multicolumn{2}{|c|}{$5.52 \times 10^{5 b}$} \\
\hline 20 & \multicolumn{2}{|c|}{$1.17 \times 10^{-5 c}$} & & \multicolumn{2}{|c|}{$4.88 \times 10^{5 c}$} \\
\hline 30 & \multirow{2}{*}{\multicolumn{2}{|c|}{$1.08 \times 10^{-5 d}$}} & & \multirow{2}{*}{\multicolumn{2}{|c|}{$4.21 \times 10^{5 \mathrm{~d}}$}} \\
\hline 40 & & & & & \\
\hline
\end{tabular}

$a, b, c, d, e$ Different superscripts indicate a statistically significant difference $(p<0.05)$

From Fig. 3d, the $10 \mathrm{wt} \% \mathrm{BP} /$ starch film exhibited the lowest $\Delta E^{*}$ value among other films, with an average value of 11.0. Average values for $\Delta E$ increased from 11.0 to 35.6 with respect to neat starch film with the increase in percentage composition of BP from 10 to $40 \mathrm{wt} \%$ consistent with the findings of $L^{*}, a^{*}$, and $b^{*}$ values. The differences might be related to the relative amount of the BP and the presence of small brown spots from BP that resulted in the cloudy effect.

Meanwhile, transparency analysis was performed using light transmittance values in the visible region at $600 \mathrm{~nm}$. The transmittance values are presented in Fig. 3e. The neat starch film was found to be the most transparent, measuring the highest transmittance value of $75.9 \%$ in the visible region. Lee et al. (2008) revealed that the transparency of films can be influenced by homogeneity and the quantity of addition of alternative biopolymer. The addition of another biopolymer would turn the control film cloudier as a result of blending the pure film with another biopolymer powder (Talja et al. 2007). From Fig. 3e, the transparency of the films seemed to decrease with the increase in BP composition due to the higher solids content in the film, which resulted in lower transparency. This was because the large amount of BP content in the starch matrix led to a blocking effect on the virtually transparent starch film in the visible spectra region (Lee et al. 2008). The reduction in the transmittance might protect the packaged foods from visible and ultraviolet light that might cause discoloration, nutrient loss, and off-flavor (Arfat et al. 2017). It is worth noting that with the increase in BP powder composition, the films became darker, and thus exhibited lower transparency, due to the original color of BP added into the films. 


\section{Mechanical Properties}

The effect of addition of different percentage composition of BP on mechanical properties of starch films is shown in Figs. $4 \mathrm{a}, \mathrm{b}, \mathrm{c}$ and tabulated in Table 1. Tensile strength is an indication of the film strength (Javadian et al. 2014); thus it is important to be determined for food application. The effect of addition of different percentage compositions of BP on TS of starch films can be seen from Fig. 4a.
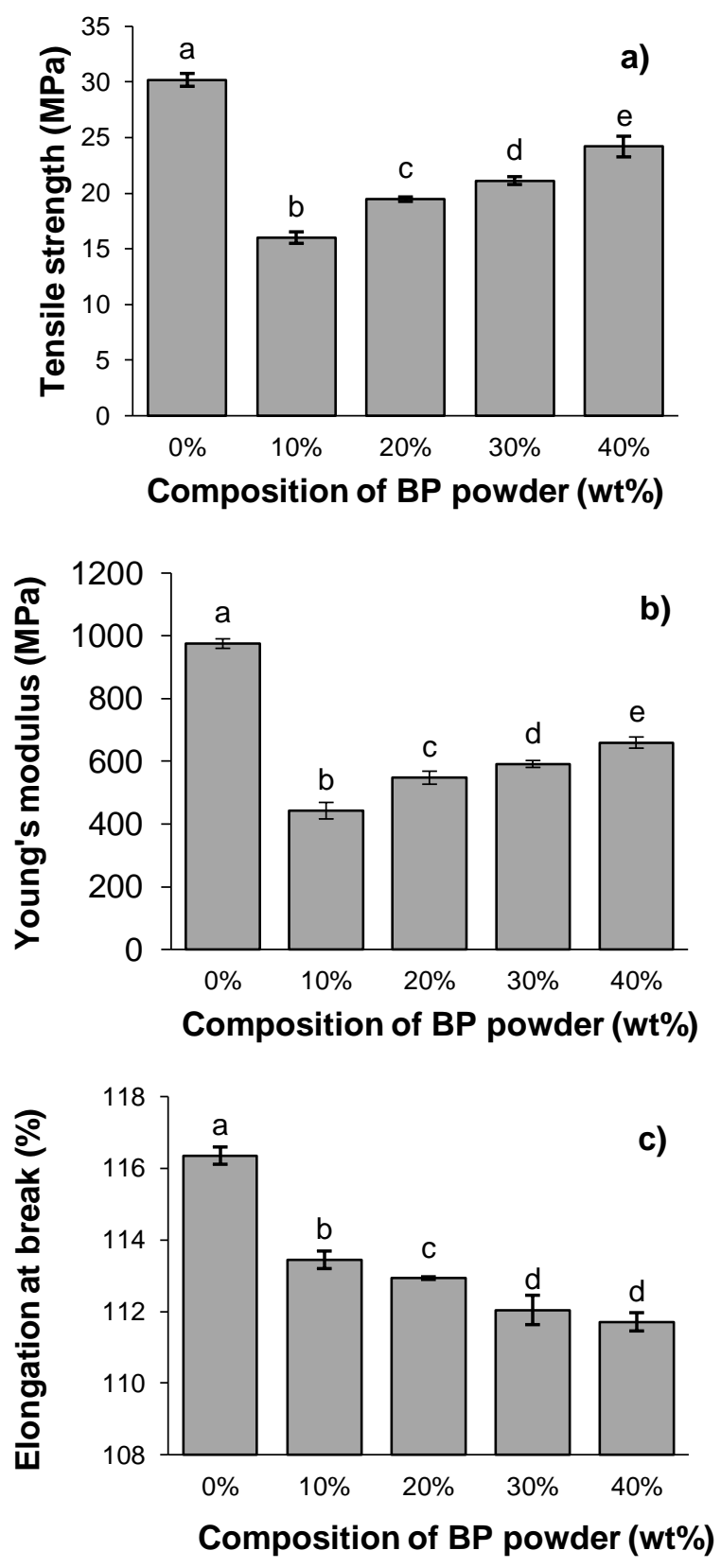

Fig. 4. Effects of addition of different percentage compositions of BP powder on (a) tensile strength, (b) Young's modulus, and (c) elongation at break of starch films. Different letters indicate a statistically significant difference $(p<0.05)$.

The neat starch film exhibited the highest TS value of $30 \mathrm{MPa}$. This was due to the strong intermolecular hydrogen bonds formed among the starch molecules. The high value 
of TS indicates brittle and rigid characteristics of the neat starch films. The value of TS decreased with the addition of $10 \mathrm{wt} \% \mathrm{BP}$ by approximately $47 \%$, thus indicating the reduction of film strength. The decrease in mechanical strength of the films was attributed to the weak molecular interaction between the starch biopolymer and BP. The decrement in TS values indicated that the films became less brittle and rigid with the incorporation of BP. Orsuwan et al. (2016) also reported that addition of banana powder into agar films resulted in the reduction of the strength of blended films due to the weak molecular interaction between the banana powder and agar matrix. The weak molecular interaction of BP and starch was due to the phase separation between the two components (Shapi'i et al. 2016), whereby strong intermolecular bond between both components was difficult to form due to insufficient concentration of BP.

Nonetheless, increasing the percentage composition of BP in the starch films led to the improvement of film strength. The TS values increased from 16 to $24 \mathrm{MPa}$ with the increase in BP composition from 10 to $40 \mathrm{wt} \%$. This trend was presumably induced by the physical interaction between starch and BP. This was due to the presence of cellulose in BP powder that acted as reinforcement in the starch matrix. Starch and cellulose both contain 1-4 glycosidic bonds, but the amylopectin portion of starch also contains 1-6 glycosidic bonds for branching. These chemical similarities induced a strong interaction between cellulose and the starch matrix through hydrogen bonding, thus improving the properties of the starch films. However, the TS value of $40 \mathrm{wt} \% \mathrm{starch} / \mathrm{BP}$ was still lower than neat starch film.

Figure $4 \mathrm{~b}$ shows the effect of addition of different percentage compositions of BP on YM of starch films. Young's modulus is a good indication of the stiffness of the film. Based on the figure, the value of YM of neat starch film decreased from 975 to $393 \mathrm{MPa}$, which was approximately a $60 \%$ decrease with the addition of $40 \mathrm{wt} \% \mathrm{BP}$. Low YM values indicated low stiffness of the film and that the film was able to withstand deformation in response to an applied force. Conversely, the higher values of YM for the neat starch film indicated that the neat starch film exhibited brittle characteristics, was easy to break, and was difficult to handle. This result demonstrated that films added with BP were less rigid and resistant to external force consistent to the result for TS. However, there was a trend of increasing YM with the increase in BP composition that demonstrated the stiffness increased with the increase in BP content. This finding was consistent with the results from the previous TS values whereby increase in TS led to an increase in the stiffness of the films, thus increasing the YM.

Elasticity of the film was determined by quantifying the EAB parameter. Figure 4c shows the EAB values of starch films incorporated with different percentage compositions of BP powder. The EAB values decreased slightly with the addition of BP in the film. The EAB values decreased from $116 \%$ to $112 \%$ with the addition of $40 \mathrm{wt} \% \mathrm{BP}$. According to Bangyekan et al. (2006), the decrease in elongation was due to the interaction between starch and cellulose occurring at the molecular level, which led to the strong adhesion between the biopolymers. The decrease in EAB value is an indication of the increase in the brittleness of the film (Voon et al. 2010); thus there was a reciprocal trend in the TS values (Fig. 4a). The mechanical properties of the composite films have been reported to be highly dependent on the interfacial interaction between the biopolymers (Ma et al. 2008). According to Voon et al. (2010), a decrease in EAB is desirable and advantageous for food packaging as it is directly related to the biodegradability of the film. When particles are embedded in a network of starch polymeric chains in the matrix, the structural rigidity or 
brittleness of the biocomposite films usually increased. Thus, a higher rigidity and brittleness of the films resulted in a lower EAB value.

It is worth noting that the films added with BP composition of higher than $40 \mathrm{wt} \%$ were excessively brittle and that the film did not form well. This was attributed to the high amount of fiber that originated from BP. Therefore, the mechanical properties of starch films added with BP at composition greater than $40 \mathrm{wt} \%$ could not be determined. It can be deduced that $40 \mathrm{wt} \% \mathrm{BP}$ was the highest amount of BP that could be added to produce a starch/BP film to best minimize BP waste. The $40 \mathrm{wt} \% \mathrm{starch} / \mathrm{BP}$ film exhibited the best mechanical properties compared to films produced with other compositions of BP and starch. In addition, the range of TS and EAB of $40 \mathrm{wt} \%$ starch/BP film still falls within that of common food packaging material such as low-density polyethylene (TS: 8.3 to 31.4 $\mathrm{MPa}$ and EAB: 100 to $650 \%$ ) and high-density polyethylene (TS: 22.1 to $31.0 \mathrm{MPa}$ and EAB: 10 to $1200 \%$ ) (Chin 2010), indicating that the film has the potential to be used as food packaging material.

\section{Barrier Properties}

Water vapor and oxygen barrier properties for food grade packaging films are essential to protect the product from moisture and gasses that can lead to microorganism growth. These properties are important to investigate to prevent or reduce moisture and gas transfer amid the food and surrounding. In this case, water vapor permeability and oxygen permeability of the produced films should be as low as possible.

The effect of addition of different percentage compositions of BP powder on WVP and OP of the starch films is shown in Figs. 5a and 5b, as well as Table 1. The WVP of starch-based films decreased with the addition of BP. The WVP value of neat starch film was $1.83 \times 10^{-5} \mathrm{~g} . \mathrm{m} / \mathrm{m}^{2} . \mathrm{kPa}$.h. The value decreased approximately $42 \%$ with the addition of $40 \mathrm{wt} \% \mathrm{BP}$ into the starch film. The WVP of the films tended to slightly decrease with the increase in BP composition. The WVP was reduced due to the formation of a rigid hydrogen bond network of cellulose from BP in the composite that was governed by a percolation mechanism (Voon et al. 2010).

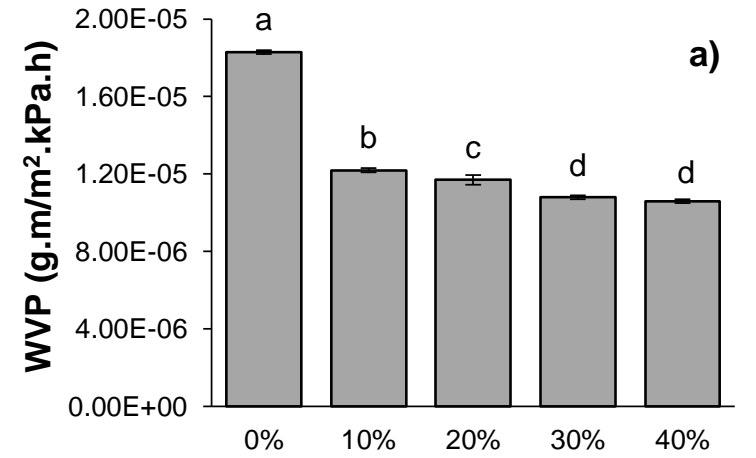

Composition of BP powder (wt\%)

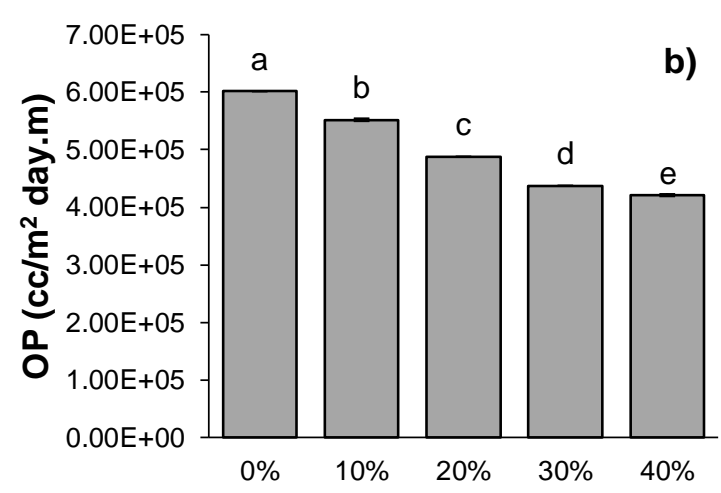

Composition of BP powder (wt\%)

Fig. 5. Effects of addition of different percentage compositions of BP powder on (a) water vapor permeability and (b) oxygen permeability of starch films. Different letters indicate a statistically significant difference $(p<0.05)$.

The improvement of the water vapor barrier of the films was attributed to the fact that the diffusion of water vapor became slower with the addition of BP powder because 
the diffusion process was influenced by the presence of impermeable particles that increased tortuous path in the film matrix (Sanchez-Garcia et al. 2008). Water molecules need to go through the tortuous path in the starch film, thus reducing the WVP.

Following this line of reasoning for food packaging application, the films usually must exhibit low oxygen barrier permeability to prevent or decrease oxidative processes during the transport, handling, and storage of the food product. The OP of the packaging film is an important characteristic to be considered for a finished packing material to increase the shelf life of foods (Miller and Krochta 1997).

Figure $5 \mathrm{~b}$ shows the effect of addition of different percentage compositions of BP powder on the OP of the starch films. The OP value of neat starch film was $6.01 \times 10^{5}$ $\mathrm{cc} / \mathrm{m}^{2}$.day. Addition of BP in the starch films reduced the OP of the starch film. A higher amount of BP added into the starch film resulted in a lower amount of oxygen that could pass through the film. The OP value decreased approximately $30 \%$ with the addition of 40 wt $\%$ BP into the starch film. Addition of BP decreased the OP of the films due to the high number of BP particles in the starch films that worked to allow less oxygen to pass through the film at $40 \mathrm{wt} \%$ of BP. The permeation of gas molecules was favored at the area between the starch and BP particles. Gas molecules were constrained to diffuse in a random path instead of taking a straight, linear path perpendicular to the film surface due to the existence of impermeable BP.

It is worth noting that the state of dispersion of BP in the starch film also affected the permeability of the films. The reduction in WVP and OP of starch films with the addition of BP was due to the generation of a more winding path for the permeation of water vapor and oxygen molecules due to the presence of well-dispersed BP in the starch biopolymer matrix (Voon et al. 2010). Thus, diffusion of the water vapor and oxygen into the film decreased relative to the amount and orientation of the BP in the matrix. It is worth noting that the state of aggregation and dispersion of BP in the film matrix are important aspects that can influence permeability of the film (Bharadwaj 2001). An increase in both the water vapor and gas barrier properties was dependent on the concept of tortuous path of the material.

\section{CONCLUSIONS}

1. The addition of BP reduced the optical and mechanical properties of the starch films. However, both the mechanical and barrier properties of the films improved with the increase in percentage composition of BP. Barrier properties of starch/BP films were significantly higher than the neat starch film.

2. It was found that starch film incorporated with $40 \mathrm{wt} \% \mathrm{BP}$ powder was the best composition to utilize the BP waste and that the $40 \mathrm{wt} \%$ starch/BP film exhibited good mechanical and barrier properties.

3. Starch/BP films produced in this study have the potential to be used as food packaging material to replace the nonbiodegradable packaging, as their mechanical properties are fall within the range of common food packaging materials. 


\section{ACKNOWLEDGMENTS}

The authors are grateful for the support of the Putra Grant - Putra Graduate Initiative (IPS), Universiti Putra Malaysia, Malaysia (Vote no. 9546200).

\section{REFERENCES CITED}

Arfat, Y. A., Ahmed, J., Hiremath, N., Auras, A., and Joseph, A. (2017). "Thermomechanical, rheological, structural and antimicrobial properties of bionanocomposite films based on fish skin gelatin and silver copper nanoparticles," Food Hydrocolloids 62, 191-202. DOI: 10.1016/j.foodhyd.2016.08.009

ASTM D1746 (2015). "Standard test method for transparency of plastic sheeting," ASTM International, West Conshohocken, PA, USA.

ASTM D882 (2018). "Standard test method for tensile properties of thin plastic sheeting," ASTM International, West Conshohocken, PA, USA.

ASTM F1927 (2014). "Standard test method determination of oxygen gas transmission rate, permeability and permeance at controlled relative humidity through barrier materials using a coulometric detector," ASTM International, West Conshohocken, PA, USA.

Bangyekan, C., Aht-Ong, D., and Srikulkit, K. (2006). "Preparation and properties evaluation of chitosan-coated cassava starch films," Carbohydrate Polymers 63(1), 61-71. DOI: 10.1016/j.carbpol.2005.07.032

Bharadwaj, R. K. (2001). "Modeling the barrier properties of polymer-layered silicate nanocomposites," Macromolecules 34(26), 9189-9192. DOI: 10.1021/ma010780b

Cheng, G., Zhou, M., Wei, Y. J., Cheng, F., and Zhu, P. X. (2019). "Comparison of mechanical reinforcement effects of cellulose nanocrystal, cellulose nanofiber, and microfibrillated cellulose in starch composites," Polymer Composites 40(S1), E365E372. DOI: $10.1002 / p c .24685$

Chin, A. W. (2010). "Polymers for innovative food packaging," Worcester Polytechnic Institute: Electronic Projects Collection, (https://web.wpi.edu/Pubs/Eproject/Available/E-project-050610124822/unrestricted/Polymers_for_Innovative_Food_Packaging.pdf), Accessed 15 July 2019.

Faradilla, R. F. H., Lee, G., Arns, J. Y., Roberts, J., Martens, P., Stenzel, M. H., and Arcot, J. (2017). "Characteristics of a free-standing film from banana pseudostem nanocellulose generated from TEMPO-mediated oxidation," Carbohydrate Polymers 174, 1156-1163. DOI: 10.1016/j.carbpol.2017.07.025

Ferracane, J. L., Pfeifer, C. S., and Hilton, T. J. (2014). "Microstructural features of current resin composite materials," Current Oral Health Reports 1(4), 205-212. DOI: 10.1007/s40496-014-0029-4

Hanpin, L., and Stephen, W. H. (2013). "Plasticizers effects on physical-mechanical properties of solvent cast soluplus films," AAPS Pharmacy Science and Technology 14(3), 903-910. DOI: 10.1208/s12249-013-9971-z

Javadian, N., Mirzai, H., and Nafchi, A. M. (2014). "The effects of ribose on mechanical and physicochemical properties of cold water fish gelatin films," Journal of Chemical Health Risks 4(2), 39-45. DOI: 10.22034/JCHR.2018.544065

Ji, N., Liu, C., Zhang, S., Yu, J., Xiong, L., and Sun, Q. (2017). "Effects of chitin nano- 
whiskers on the gelatinization and retrogradation of maize and potato starches," Food Chemistry 214, 543-549. DOI: 10.1016/j.foodchem.2016.07.113

Jiménez, A., Fabra, M. J., Talens, P., and Chiralt, A. (2012). "Edible and biodegradable starch films: A review," Food and Bioprocess Technology 5(6), 2058-2076. DOI: 10.1007/s11947-012-0835-4

JIS Z 0208 (2017). "Testing methods for determination of the water vapour transmission rate of moisture-proof packaging materials (dish method)," Japanese Standards Association, Tokyo, Japan.

Lee, D. S., Yam, K. L., and Piergiovanni, L. (2008). Food Packaging Science and Technology, CRC Press, Boca Raton, FL, USA.

Ma, X., Chang, P. R., and Yu, J. (2008). "Properties of biodegradable thermoplastic pea starch/carboxymethyl cellulose and pea starch/microcrystalline cellulose composites," Carbohydrate Polymers 72(3), 369-375. DOI: 10.1016/j.carbpol.2007.09.002

Miller, K. S., and Krochta, J. M. (1997). "Oxygen and aroma barrier properties of edible films: A review," Trends in Food Science and Technology 8(7), 228-237. DOI: 10.1016/S0924-2244(97)01051-0

Nordin, N., Othman, S. H., Kadir Basha, R., and Abdul Rashid, S. (2018). "Mechanical and thermal properties of starch films reinforced with microcellulose fibres," Food Research 2(6), 555-563. DOI: 10.26656/fr.2017.2(6).110

Orsuwan, A., Shankar, S., Wang, L. F., Sothornvit, R., and Rhim, J. W. (2016). "Preparation of antimicrobial agar/banana powder blend films reinforced with silver nanoparticles," Food Hydrocolloids 60, 476-485. DOI: 10.1016/j.foodhyd.2016.04.017

Othman, S. H. (2014). "Bio-nanocomposite materials for food packaging applications: Types of biopolymer and nano-sized filler," Agriculture and Agricultural Science Procedia 2, 296-303. DOI: 10.1016/j.aaspro.2014.11.042

Othman, S. H., Kechik, N. R. A., Shapi'i, R. A., Talib, R. A., and Tawakkal, I. S. M. A. (2019a). "Water sorption and mechanical properties of starch/chitosan nanoparticle films," Journal of Nanomaterials Article ID 3843949. DOI: 10.1155/2019/3843949

Othman, S. H., Majid, N. A., Tawakkal, I. S. M. A., Kadir Basha, R., Nordin, N., and Shapi'i, R. A. (2019b). "Tapioca starch films reinforced with microcrystalline cellulose for potential food packaging application," Food Science and Technology 39(3), 605-612. DOI: 10.1590/fst.36017

Pacaphol, K., Seraypheap, K., and Aht-Ong, D. (2019). "Development and application of nanofibrillated cellulose coating for shelf life extension of fresh-cut vegetable during postharvest storage," Carbohydrate Polymers 224(July), 115167. DOI: 10.1016/j.carbpol.2019.115167

Pappu, A., Patil, V., Jain, S., Mahindrakar, A., Haque, R., and Thakur, V. K. (2015). "Advances in industrial prospective of cellulosic macromolecules enriched banana biofibre resources: A review," International Journal of Biological Macromolecules 79, 449-458. DOI: 10.1016/j.ijbiomac.2015.05.013

Resa, C. P. O., Gerschenson, L. N., and Jagus, R. J. (2012). "Effect of natamycin on physical properties of starch edible films and their effect on Saccharomyces cerevisiae activity," Food and Bioprocess Technology 6(11), 3124-3133. DOI: 10.1007/s11947-012-0960-0

Sanchez-Garcia, M. D., Gimenez, E., and Lagaron, J. M. (2008). "Morphology and barrier properties of solvent cast composites of thermoplastic biopolymers and purified cellulose fibers," Carbohydrate Polymers 71(2), 235-244. DOI: 
10.1016/j.carbpol.2007.05.041

Shapi'i, R. A., and Othman, S. H. (2016). "Effect of concentration of chitosan on the mechanical, morphological and optical properties of tapioca starch film," International Food Research Journal 23(December) 187-193.

Shapi'i, R. A., Othman, S. H., Nazli Naim, M., and Kadir Basha, R. (2019). "Mechanical properties of tapioca starch-based film incorporated with bulk chitosan and chitosan nanoparticle: A comparative study," Pertanika J. of Sci. and Technol. 27, 95-107.

Talja, R. A., Helén, H., Roos, Y. H., and Jouppila, K. (2007). "Effect of various polyols and polyol contents on physical and mechanical properties of potato starch-based films," Carbohydrate Polymers 67(3), 288-295. DOI: 10.1016/j.carbpol.2006.05.019

Tibolla, H., Pelissari, F. M., Martins, J. T., Lanzoni, E. M., Vicente, A. A., Menegalli, F. C., and Cunha, R. L. (2019). "Banana starch nanocomposite with cellulose nanofibers isolated from banana peel by enzymatic treatment: In vitro cytotoxicity assessment," Carbohydrate Polymers 207(November), 169-179. DOI: 10.1016/j.carbpol.2018.11.079

Vásconez, M. B., Flores, S. K., Campos, C. A., Alvarado, J., and Gerschenson, L. N. (2009). "Antimicrobial activity and physical properties of chitosan-tapioca starch based edible films and coatings," Food Research International 42(7), 762-769. DOI: 10.1016/j.foodres.2009.02.026

Voon, H. C., Bhat, R., Easa, A. M., Liong, M. T., and Karim, A. A. (2010). "Effect of addition of halloysite nanoclay and $\mathrm{SiO}_{2}$ nanoparticles on barrier and mechanical properties of bovine gelatin films," Food and Bioprocess Technology 5(5), 17661774. DOI: 10.1007/s11947-010-0461-y

Wang, W., Yu, Z., Alsammarraie, F. K., Kong, F., Lin, M., and Mustapha, A. (2020). "Properties and antimicrobial activity of polyvinyl alcohol-modified bacterial nanocellulose packaging films incorporated with silver nanoparticles," Food Hydrocolloids 100(2020), Article ID 105411. DOI: 10.1016/j.foodhyd.2019.105411

Wheatley, C. C. (2003). "Cassava: The nature of the tuber," in: Encyclopedia of Food Sciences and Nutrition, 2nd Ed., L. C. T. B. Caballero, and P. M. Finglas (eds.), Academic Press, Elsevier Science, London, England, pp. 969-969. DOI: 10.1016/B012-227055-X/00181-4

Wittaya, T. (2009). "Microcomposites of rice starch film reinforced with microcrystalline cellulose from palm pressed fiber," International Food Research J. 16(4), 493-500.

Zahiruddin, S. M. M., Othman, S. H., Tawakkal, I. S. M. A., and Talib, R. A. (2019). "Mechanical and thermal properties of tapioca starch films plasticized with glycerol and sorbitol," Food Research 3(2), 157-163. DOI: 10.26656/fr.2017.3(2).105

Zainuddin, S. Y. C., Ahmad, I., Kargarzadeh, H., Abdullah, I., and Dufresne, A. (2013). "Potential of using multiscale kenaf fibers as reinforcing filler in cassava starchkenaf biocomposites," Carbohydrate Polymers 92(2), 2299-2305. DOI: 10.1016/j.carbpol.2012.11.106

Zhang, R., Wang, X., and Cheng, M. (2018). "Preparation and characterization of potato starch film with various size of nano-SiO 2 ," Polymers 10(10), Article Number 1172. DOI: $10.3390 /$ polym10101172

Article submitted: November 4, 2019; Peer review completed: March 8, 2020; Revised version received and accepted: March 31, 2020; Published: April 8, 2020.

DOI: 10.15376/biores.15.2.3984-3998 